\title{
Optimal Design of Inventory Management Systems for Micro-Warehousing in the Healthcare Industry
}

\author{
B. Guo, R. García-Flores and M. Ayre ${ }^{a}$ \\ ${ }^{a}$ CSIRO Digital Productivity Flagship, Private Bag 33, Clayton South, VIC, Australia, 3169 \\ Email: Rodolfo.Garcia-Flores@csiro.au
}

\begin{abstract}
Reverse logistics' research has focused on how to improve current operations to recover some value from supply chains that distribute and sell products that end up with a customer. For high-value, low-volume supply chains, particularly those operating on a contract-loan basis such as specialized medical goods, reverse logistics has a different value proposition. As with most unidirectional supply chains, these have developed over time out of necessity and without a plan or design to operate efficiently. As health care has become widespread and universal, they have grown organically into systems that are far from optimal.

In contrast to traditional supply chains, chains working on loan contracts are inherently closed, as products must be returned for re-fitting and conditioning before the next loan, and thus any inefficiencies in reverse logistics will directly impact the business. Moreover, at the consumer end of the health care sector (that is, the hospitals), efficiency for the supplier is not necessarily seen as a priority. In this paper, we investigate two important logistic questions that distributors of goods for the health industry face today. First, what are the efficiency gains if the medical good distributor assumes the lead role in centralising some operations (e.g., in sterilisation policies) which are currently distributed and owned by health staff? Second, can new sensors and the Internet of Things (IoT) assist reverse logistics of medical material in improving services, lead times and bottom lines of hospitals and distributors, without impacting patient care? We introduce an optimisation model to calculate an optimal distribution schedule to address these questions.
\end{abstract}

Keywords: Inventory management, health care, logistics for the health industry, optimisation, mixed-integer linear programming 


\section{INTRODUCTION}

According to health care industry surveys [Motorola Inc. [2010], the costs of managing the supply chain are second only to the costs of labour. This source indicates that supply chain management consumes between one third and two thirds of the annual operating costs for health care providers, distributors, group purchase organisations and manufacturers; inventory management and order management alone represent approximately half of those costs. Despite the importance of health care logistics, the industry remains mired in outdated business practices and processes, lack of standards, poor collaboration among suppliers, distributors and hospital and clinic managers, while at the same time it faces growing economic pressure from stakeholders, increasing demands from an aging population, and unplanned complexity produced by building upon existing and inefficient legacy operations.

Awareness of these problems is widespread, and a number of overviews exist that summarise the most pressing issues. Rossetti et al. [2008] identifies opportunities for applications of inventory management to the health care value chain, with emphasis in demand forecasting, just-in-time and multi-objective optimisation. The overview given in Chandra and Kachhal [2004] indicates that the most common problems include the configuration of distribution networks, inventory control, supply contracts, distribution strategies, supply chain integration and strategic partnering, outsourcing and procurement strategies, information technology and decision support systems, and customer value. Many of these logistic challenges stem from the health industry's fragmented supplier base, legacy inefficiencies, increasing cost of materials, and decreasing resources and subsidies.

In this paper, we focus on the challenge of reverse logistics for distribution networks of high value-added medical products. Reverse logistics is the process of planning and controlling the efficient and cost-effective flow of materials, in-process inventory and finished goods from the point of consumption to the point of origin for the purpose of recapturing value or proper disposal [Govindan et al., 2015]. We will use as an example the consignment and loan of orthopaedic kits, but the following reasoning applies just as well to equipment used in other medical specialties. A major supplier of orthopaedic products would handle tens of thousands of stock-keeping units (SKUs or kits), distribute them to hundreds of hospitals and hold hundreds of millions of dollars in inventory. The kit contains trays of instruments, which are re-usable, and implants, which are consumable. These products are not sterilised, but the trays do include articles that are sterilised; trays are designed to facilitate sterilisation. Many consumables have expiry dates, thus requiring stock rotation. New products are often released, making it necessary to plan for the retirement of kits. In addition to this, many of the re-usable components need to return to the distributor for fitting and re-conditioning, and the longer the period of time the hospitals hold to these instruments after use, the more of an inconvenience it is to all the involved parties, as the kit is not available for re-distribution to another hospital. Unfortunately, at present it is not possible to track the location of individual kits with accuracy. These features make it clear that the supply chain does not end once the kit is delivered to a customer, but a reverse logistics operation is crucial.

Not many projects deal with reverse logistics in the health industry. Some examples include Li-Hong [2009], who focuses on the problem of recovering medical waste, which has important public health and environmental implications. The reverse logistics model proposed calculates the optimal locations of collection and processing centres. The model proposed finds the optimal location of collection and processing centres. Similar models to the one we propose here, although not related to the health industry, include Krikke et al. [1999], who optimise the design of a reverse logistic network for a photocopier manufacturer. Like the orthopaedic kits, photocopiers are complex items with some valuable parts used in re-manufacturing, some others for refurbishment and some others to disposal. Li [2013] proposes a probability-based triple bottom line framework for assessing reverse supply chains that takes into consideration costs, product quality and environmental harm.

In the present paper, we introduce a reverse logistics model with the objectives of, first, increasing the logistic efficiency of the medical kits' distribution network, and second, to make the best possible use of the kits by minimising the idle time the equipment spends in the hospitals after use. We argue that the proposed model could be used in conjunction with improvements in day-to-day operations such as real-time automated data capture to keep track of the medical kits within the system, and that the model could assist in assessing existing and proposed standards in the current supply chain configuration. For example, by defining policies for sterilisation at the companies' warehouses rather than having kits sterilised at hospitals as well, could lead to important potential savings. 


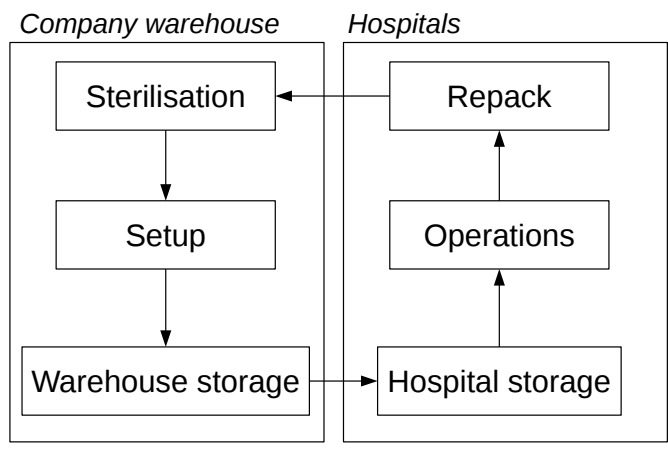

(a) Closed-loop supply chain structure

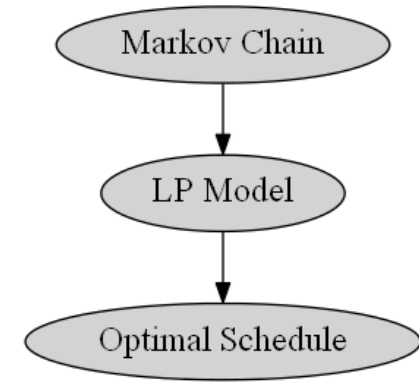

(b) Schematic of model relationship

Figure 1: The reverse health logistics supply chain and the relationship among model components

\section{The Medical Kit Supply Chain}

The schematic in Figure 1a highlights the main operations and will form the basis of our model. After preparation, the kits flow from warehouse storage to hospital storage, where it remains for a variable amount of time. Once the kits are used in medical operations, they are then reassembled, sterilised and prepared for storage before being sent back to the company's warehouse. The standard hospital procedure is to sterilise the kits prior to operation; however, only one sterilisation step is rationally required in the supply chain model. Due to a lack of agreed standards across the industry, hospitals are apprehensive of whether the sterilisation at the company's warehouse meets their own standards. For our model, we shall assume that such a standard exists and only one sterilisation happens at the company's warehouse.

Some aspects of the system, such as the inclusion of manufacturer and end disposal, were left out for simplicity reasons. These assumptions tend to hold true in short, operational time frames, but in the long run, strategic analysis would also need to consider these components of the supply chain. The linear model we present optimises network flows and inventories simultaneously. The decision variables represent the schedule of deliveries from warehouses to hospitals and the return of materials in the opposite direction. The model will let us address vital questions such as what is the best schedule with known demand? Where should certain operations (such as warehousing) be done to minimise cost? and What should be the baseline inventory stock level? In the following section, we present a linear program (LP) for optimising scheduling in order to minimise operational costs. The solution prescribes the transportation path and quantity that will minimise the aforementioned costs for the company and the consumers.

\section{The Mathematical Model}

In what follows, $L$ refers to the set all warehouse and hospital locations, $K$ to the set of all kit types, $G$ to the set of possible 'ages' (shifts held at hospital) of the kits, and $T$ to the set of all time shifts considered. The time horizon of the optimisation consists of 60 shifts. Our decision variables are $x_{i j g k t}$, the flow of kits of type $k$ between $i$ and $j$ which have been held for $g$ shifts at shift $t ; p_{i k t}$ is the inventory size at location $i$ for kit type $k$ and at the time shift $t$; $q_{i g k t}$ represents the inventory size of kits of age $g$; and $s_{k i t}$ is the total unsatisfied demand of kit type $k$ at hospital $i$ in shift $t$.

We modelled the variability of the time the hospitals hold the kits using a Markov Chain (MC) by representing the probabilities of a kit passing to different stages of the supply chain (explained below). The solution prescribes the transportation path and quantity that will minimise the aforementioned costs for the company and the consumers. Figure $1 \mathrm{~b}$ illustrates how the MC model's results are used as parameters in the LP model. The aim of the problem is to minimise the sum of transportation, inventory holding, kit setup, timing penalty and unsatisfied demand costs:

$$
\begin{aligned}
\min & \sum_{(i j) \in L} T C_{i j} \sum_{g \in G} \sum_{k \in K} \sum_{t \in T} x_{i j g k t}+\sum_{k \in K} \sum_{i \in L} Q C_{i k} \sum_{t \in T} p_{i k t}+\sum_{i \in W} \sum_{k \in K} S C_{i k} \sum_{j \in H} \sum_{t \in T} x_{j i, 0, k t}+ \\
& \sum_{k \in K} P C_{k} \sum_{g>\tau(g)} \sum_{-\rho} \sum_{i \in H} q_{i g k t}+\sum_{k \in K} D C_{k} \sum_{i \in H} \sum_{t \in T} s_{k i t},
\end{aligned}
$$


where $T C_{i j}$ is the transportation cost between location $(i, j) \in J$ per kit, $Q C_{i k}$ is the inventory holding cost between for kit $k \in K$ at location $i \in L, S C_{i k}$ is the setup cost for kit $k \in K$ at warehouse $i \in W, P C_{k}$ is the penalty cost for not timely returning kit $k \in K$ to a warehouse and $D C_{k}$ is the unsatisfied demand cost for a kit $k \in K ; \tau$ and $\rho$ represent the estimated periods on hold and the time in which the kit is used and are estimated beforehand using a normal distribution, although they could be expressed as a function of $g$. We next outline the most important constraints.

Variable $p_{i k t}$ is defined as

$$
p_{i k t}=\sum_{g \in G} q_{i g k t} \quad \forall i \in L, \forall k \in K, \forall t \in T
$$

where $G$ represents the time that a kit has remained in a hospital.

For cases where the kits arrive to or depart from hospitals, we have

$$
\sum_{j \in W} x_{j i g k t}-\sum_{j \in W} x_{i j g k t}=q_{i g k t}-q_{i, g-1, k, t-1} \quad \forall i \in H, \forall g \in G \backslash\{0\}, \forall k \in K, \forall t \in T \backslash\{0\}
$$

which says that the number of kits that enters a hospital is equal to the amount of kits that accumulates in the inventory during that period. Additionally, the age $g$ of the kits located in hospitals are incremented at each time shift. The indexes associated with constraint (3) specify that medical kits are only delivered to hospitals from warehouses, $W$, and are sent back to warehouses, $W$, once their use is ceased. There is an analogous constraint to model flows to and from the warehouses, not shown due to space limitations.

Once the kits are sent back to the warehouses, they must undergo maintenance checks before being repackaged. It is assumed that such a process must take at least one time shift. Constraint

$$
\sum_{j \in L} x_{i j, 0, k t} \leq q_{i, 0, k, t-1} \quad \forall i \in W, \forall g \in G, \forall k \in K, \forall t \in T \backslash\{0\} .
$$

states that only kits currently present in the warehouse's inventory can be sent to other locations. The model also states that return kits cannot be immediately sent to a new location, but must remain at the warehouse for at least one shift.

Kits sent to the hospitals together must return together. Constraints

$$
\begin{aligned}
\sum_{j \in W} x_{j i, 0, k t} & =\sum_{j \in W} x_{i j, \tau, k, t+\tau} & & \forall i \in H, \forall k \in K, \forall t \in T, \\
q_{i, 0, k t} & =q_{i g k, t+g} & & \forall i \in H, \forall g \in G \backslash\{g \geq \tau\}, \forall k \in K, \forall t \in T,
\end{aligned}
$$

state that all kits of type $k$ that arrive at a hospital at a time, leave the hospital together $\tau$ periods later. Constraint (6) is an almost equivalent to constraint (5) but makes the explicit statement that no further flow of that order can occur while the hospital has the packages in their possession. Constraint (5) explicitly states that the kits travel together. The assumption behind these constraints is that, if the hospital has not returned the kits they currently hold, then they will not require more orders of the same type of kit.

We express the relation between transported kits and the hospitals' demand as

$$
D_{j k t}=\sum_{i \in W} x_{i j, 0, k t}+s_{j k t}-e_{j k t} \quad \forall k \in K, \quad \forall j \in H, \quad \forall t \in T,
$$

which says that for warehouses, the amount sent to the hospitals is always equal to the demand minus the unsatisfied demand $\left(s_{j k t}\right)$ plus the excess deliveries $\left(e_{j k t}\right)$. Unsatisfied demand is assumed to occur due to either a lack of inventory or a decision that proves to be more optimal. Excess deliveries are included to represent the contrary case where the total expenditure of the system can be minimised by sending kits ahead of time. Excess deliveries will also prove vital in the periodic constraints discuss further in this section.

The conservation constraints state that the number of kits of type $k$ in the system, $N_{k}$, remains constant. This assumes that no kit is lost or broken, which is not true as in reality this happens from time to time. The constraint can be expressed as

$$
\sum_{i \in L} p_{i k t}=N_{k} \quad \forall t \in T, \quad \forall k \in K
$$


B. Guo, et al., Optimal Inventory Management for the Healthcare Industry

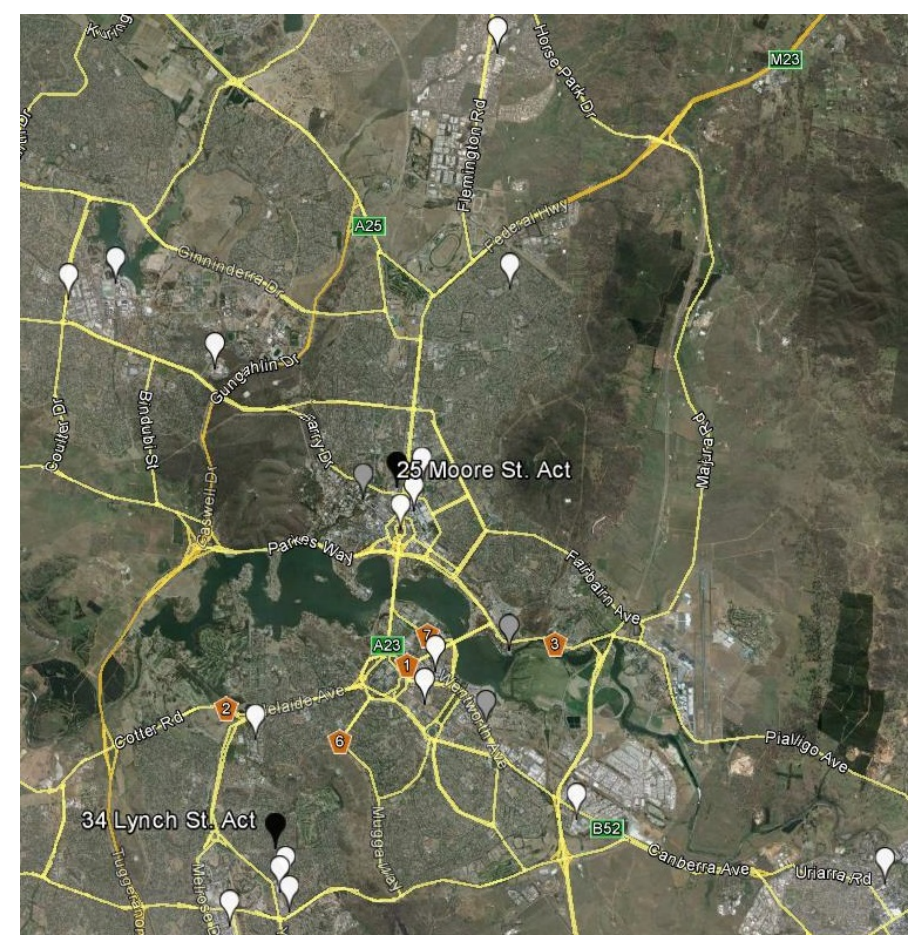

Figure 2: Locations of Canberra's hospitals (white) and warehouses (red)

To establish a cyclic pattern for inventories we set the requirement that inventory and flow are equal at the start and end of the month.

$$
\begin{aligned}
x_{i j g k t_{0}} & =x_{i j g k, t_{0}+|T|} & & \forall i \in L, \forall j \in L, \forall g \in G, \forall k \in K, \\
q_{i g k t_{0}} & =q_{i g k, t_{0}+|T|} & & \forall i \in L, \forall g \in G, \forall k \in K,
\end{aligned}
$$

where $t_{0}$ and $T$ enable the calculation of a periodic solution. To obtain the value of $\tau$ and $\rho$ in the steady state, the Markov Chain (MC) model is constructed using the probabilities in Figure 3 to find the long-term and stochastic behaviour of parameter $\tau$, the number of time shifts spent by a medical kit in a hospital during its stay.

\section{RESUlts AND Discussion}

Canberra (Figure 2) was the city chosen for the case study. Hospital locations are actual, warehouse locations are hypothetical and were selected randomly. The city features 20 main hospitals with different specialties ranging from dental to surgical. The warehouse locations selected arbitrarily are 25 Moore St and 34 Lynch St. The distances between every pair of warehouses and hospitals were calculated using the shortest possible road route to form 231 distance combinations in total. These distances where used to calculate the transportation cost using the relationship: distance $\propto$ transportation cost where the factor was defined according to the type of medical kit being delivered.

In summary, the inputs to the optimisation model are parameters $\tau$ and $\rho$, which are obtained in the preprocessing stage from a MC model, the demand in time by each hospital, the locations of hospitals and warehouses, and general costs such as transportation, kit setup, penalties, unsatisfied demand and inventory holding costs. The output is represented by the inventory, flow and unsatisfied demand decision variables.

All calculations were made using a shared remote desktop running CPLEX 12.5.0.1 in a 64-bit Intel Xeon $\mathrm{CPU}$ at $2.80 \mathrm{GHz}$ (2 processors) and 64GB of RAM. The program was solved in parallel mode using up to 23 threads for concurrent optimization. The resulting LP has 2024 constraints and 8258 variables. The total time required to solve the model was $0.5 \mathrm{~s}$ with the optimal scheduling resulting in a total cost of $\$ 754$ and $\$ 1299$ for the low and high demand scenarios, respectively. For all the results, $N=10$ for a single type of kit in the system. 


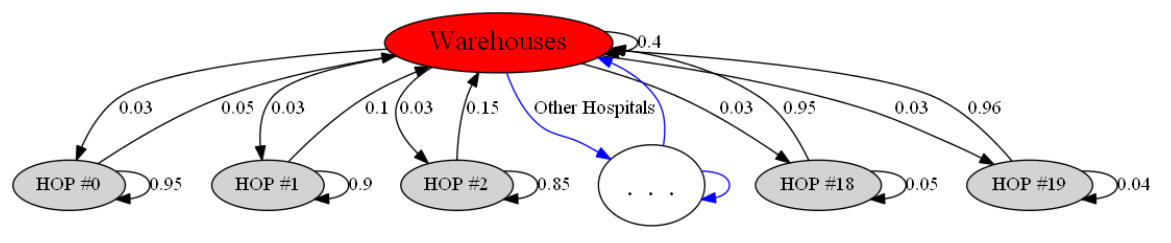

Figure 3: State diagram for the medical kit's location

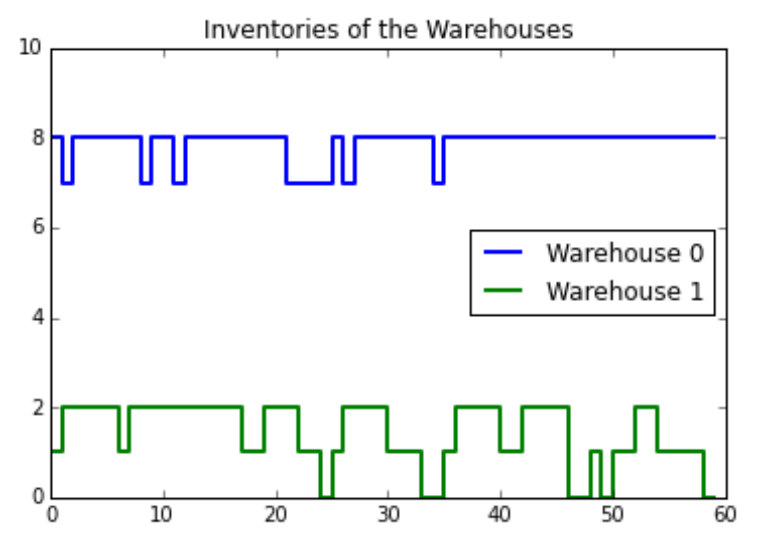

(a) The steady-state solution for low demand

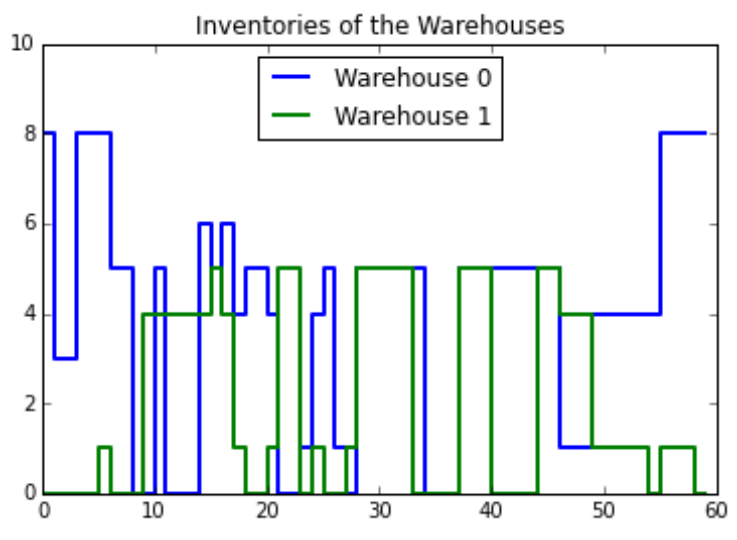

(b) The steady-state solution for high demand

Figure 4: Steady state inventories

We tested two scenarios, low and high demand, for which the inventory results are shown in Figures 4 a and $4 \mathrm{~b}$, respectively. These figures represent variations of variable $q_{i g k t}$ respect to time. The low demand model was solved using a pre-defined demand schedule (not shown due to space limitations) with a quantity of one medical kit per order. The high demand model was assuming five medical kits per order.

Comparing figures $4 \mathrm{a}$ to $4 \mathrm{~b}$ we see different behaviours of the warehouses inventories. For the low demand situation, the inventories of the warehouses remain stable with little fluctuations from its initial condition. Intuitively, we would expect inventories to remain relatively constant as the low demand introduces few perturbations into the system. In contrast, we see that the high demand case yields a more variable inventory level. This result can be interpreted as the demand outstripping supply and thus cannot remain in a equilibrium state similar to the other case. Comparing the costs that form the objective function, we found (Figure 5 that transport, set up, timing and unmet demand costs increased under higher demand. This is a results which can be understood intuitively, as a higher demand will lead to more medical kits being delivered and hence the transportation costs can be expected to increase. Greater demand also implies that some of the demand will be unsatisfied, especially if demand outstrips supply, and thus, greater penalties will have to p be paid. Setup and timing costs are also expected to increase due to the cumulative costs associated with greater flow in the closed-loop supply chain.

\section{Concluding Remarks}

Reducing the cost in the health care industry is an economically attractive proposition. In this paper, we have formulated a mathematical model that determines the optimal baseline inventories and decisions. We have demonstrated the feasibility of our approach on a hypothetical case study based on a set of 20 hospitals in Canberra and two hypothetical warehouses of a company that supplies medical kits.

Our results for the case study show that the high demand scenario yields a more variable inventory level than the low demand case. Comparing the costs that form the objective function, we also found that transport, set up, timing and unmet demand costs increased under higher demand, as expected. Inventory management costs are almost the same for both cases, but transport and the costs of unmet demand are much greater for the high demand scenario. 
B. Guo, et al., Optimal Inventory Management for the Healthcare Industry

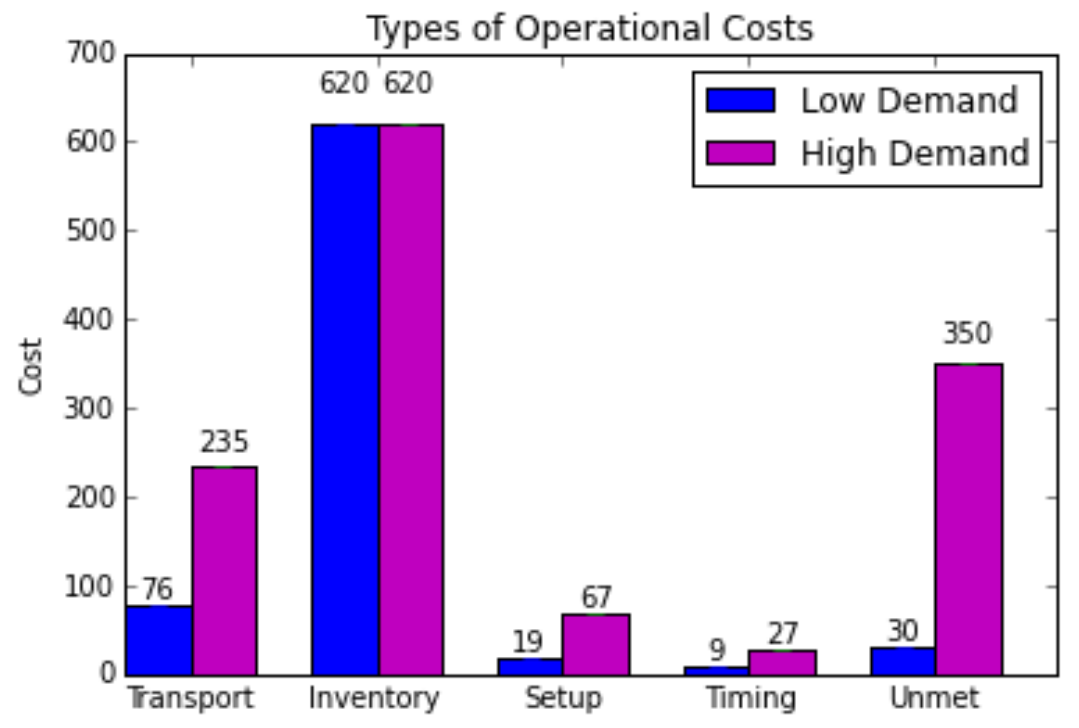

Figure 5: The relative proportions of each type of cost

The usefulness of the model we presented can be justified on the grounds of the following two problems. First, it can be used in conjunction with real-time automated data capture, obtained by installing sensors to keep track of the medical kits within the system. These sensors would add certainty to the time parameters we dealt with using Markov Chains. Second, the model could be used to assess the benefits of existing and proposed standards in the current supply chain configuration. For example, important savings could be achieved by defining clear policies and supply chain-wide standards for kit sterilisation and preparation and applying them at the companies' warehouses rather than having kits unnecessarily sterilised at hospitals for a second time. This would avoid effort duplication and save scarce and valuable resources.

\section{REFERENCES}

Chandra, C. and S. Kachhal (2004). Managing health care supply chain: trends, issues, and solutions from a logistics perspective. Proceedings of the Sixteenth Annual Society of Health Systems Management Engineering Forum. Orlando, Florida, February 20-21.

Govindan, K., H. Soleimani, and D. Kannan (2015, February). Reverse logistics and closed-loop supply chain: A comprehensive review to explore the future. European Journal of Operations Research 240(3), 603-626.

Krikke, H., A. Harten, and P. Schuur (1999, July). Business case Océ: reverse logistic network re-design for copoers. OR Spectrum 21(3), 381-409.

Li, C. (2013). An integrated approach to evaluating the production system in closed-loop supply chains. International Journal of Production Research 51(13), 4045-4069.

Li-Hong, S. (2009). A Mixed Integer Linear Programming for medical waste reverse logistics network design. Proceedings of the 16 International Conference on Management Science \& Engineering, 1971-1975. Moscow, Russia, September 14-16.

Motorola Inc. (2010). Reducing healthcare costs with supply chain best practices. how standardized data capture saves time and improves care. Technical Report IB-ISG-HCSPLYBP-0610, Motorola Inc., 1301 E. Algonquin Road, Schaumburg, Illinois 60196 U.S.A. http://www.motorolasolutions.com/web/Business/Products/_ChannelDetails/_Documents/_StaticFiles/ Healthcare-Industry-Brief-0610.pdf, accessed on January 302015.

Rossetti, M., D. Marek, S. Prabhu, A. Bhonsle, S. Sharp, and Y. Liu (2008, June). Inventory management issues in health care supply chains. Technical report, Department of Industrial Engineering, University of Arkansas, 4207 Bell Engineering Center, Fayetteville, AR 72701. http://cavern.uark.edu/rrossetti/reports/healthcare_supply_chain_rep.pdf, accessed on February 22015. 